\title{
Thermoelectric transport and spin density of graphene nanoribbons with Rashba spin-orbit interaction
}

\author{
Xinjun Cheng and Guo-Ya Sun* \\ Department of Physics, and Institute of Theoretical Physics and Astrophysics, \\ Xiamen University, Xiamen 361005, China
}

(Dated: April 25, 2016)

\begin{abstract}
In the present paper, we have theoretically investigated thermoelectric transport properties of armchair and zigzag graphene nanoribbons with Rashba spin-orbit interaction, as well as dephasing scattering processes by applying the nonequilibrium Green function method. Behaviors of electronic and thermal currents, as well as thermoelectric coefficients are studied. It is found that both electronic and thermal currents decrease, and thermoelectric properties been suppressed, with increasing strength of Rashba spin-orbit interaction. We have also studied spin split and spin density induced by Rashba spin-orbit interaction in the graphene nanoribbons.

Keywords: Graphene nanoribbons; Thermoelectric transport; Rashba spin orbit interaction; Dephasing scattering; Spin density
\end{abstract}

*Corresponding author: gysun@xmu.edu.cn (Guo-Ya Sun) 


\section{INTRODUCTION}

In recent years many efforts have been made in searching for efficient thermoelectric materials because of their potential applications in the next generation of integrated circuits[1, 2], aiming at possible conversion of waste heat into electricity. As a superstar new material, graphene has been extensively investigated and it was found that graphene[3] have excellent electrical properties due to its unique two-dimensional honeycomb structures. Using few-layers of graphene, scientists had demonstrated a metallic field-effect transistor in which the conducting channel can be switched between two-dimensional electron and hole gases by changing the gate voltage.[4,5] The success in fabricating single layer graphene has stimulated renewed extensive research efforts in graphene related research area, both theoretically and experimentally.[6] Moreover, graphene sheets have the potential to be lithographed to a lot of patterned graphene nanoribbons to make large-scale integrated circuits.

Of particular interests are currently thermoelectric properties of graphene nanoribbons (GNRs), which may exhibit an enhanced thermoelectric efficiency. Although it is known that the thermoelectric properties of a pristine graphene sheets is poor, i.e., its Seebeck coefficient is too small because of the gapless character, and consequently the figure of merit $Z T$ is low. Many experimental efforts was made to improve thermoelectric properties of graphene, with different strategies of nanostructuring as graphene nano-hole lattices[7], hy-

brid graphene/boron nitride structures [8], graphene nanoribbons with a nanopore array[9], graphene nanoribbons consisting of alternate zigzag and armchair sections [10], and graphene nanoribbons with a chevron-type geometry [11]. The main physical concerns among such 
nanostructuring lies in two aspects, one is to create gap in the electron energy bands to enhance the Seebeck coefficient, the other is to suppress the phonon conductivity. However, remarkably less theoretical work has been done up to now on thermoelectric properties of graphene, $[12,13]$ while existed theoretical works mainly focused on diffusion transport regime in graphene with impurities and other structural defects. $[14,15]$ It was reported that resonant scattering from impurities with short-range potential may lead to an enhanced Seebeck coefficient, when the chemical potential is in the neighborhood of the resonances.[16-18] In graphene, both intrinsic and extrinsic spin-orbit interactions are ready to be presented. Kane and Mele had shown that the intrinsic spin-orbit interaction can not only open a gap, but also give rise to a quantum spin Hall phase, due to localized edge states.[19, 20] The intrinsic spin-orbit interaction is quite weak due to small mass of carbon atom, and consequently small opened gap. While the extrinsic spin-orbit interaction is usually resulted from broken of inversion symmetry of the graphene lattice due to substrate electrical field, and it is known as Rashba-type spin-orbit (RSO) interaction. RSO can be tuned externally by a gate voltage and be substantially large. For example, a large Rashba-type spin-orbit interaction in epitaxial graphene grown on Ni substrate has been reported, which may reach values up to $0.2 \mathrm{eV}$.[21-23] On contrary to the intrinsic one, the extrinsic Rashba spin orbit interaction acts in the opposite direction and tends to close the gap, as well as leading to a splitting of the Dirac point into four identical points. The effects of RSO on the energy band, as well as the thermoelectric properties of graphene is interesting to be explored. One purpose of the present paper is to investigate the effect of Rashba spin orbit interaction on 
thermoelectric transport properties of graphene. Since the intrinsic spin-orbit interaction is very small, it is neglected here.

It is known that dephasing scattering processes always present because of thermal environment fluctuations, electron-electron interaction, etc. An electron continues to sustain a random fluctuating potential, which may fluctuate on a picosecond time scale. Indeed, transport in nanoscale devices is often based on the coherent evolution of the wave function and is therefore very sensible to decoherence caused by the interaction between carriers and the environment, which eventually reduces the degree of the predictability of system evolution. Therefore, it is necessary to include such dephasing processes when dealing with realistic quantum transport problems.[24] The dephasing scattering processes inside the nanoribbons can be easily accounted in the framework of the nonequilibrium Green's function (NEGF) theory, which is comparable to the Buttiker probe model[25] in conceptual and numerical simplicity, and particularly, allows one the flexibility of adjusting the degree of phase and momentum relaxation, independently.

In the present paper, we will investigate of thermoelectric transport properties of graphene, for two most popular GNRs with zigzag and armchair edges, by self-consistent atomic calculation based on NEGF theory. Concentrations are focused on effects of Rashba spin orbit interaction and dephasing scattering processes. Two specific choices of the selfenergy that provide momentum conserving and momentum relaxing dephasing processes are employed, and their different effects are obtained. The paper is organized as follows. The physics model is described in section II. Numerical results and discussion of electronic 
current, thermal current, as well as thermoelectric coefficients are presented in section III. RSO interaction induced spin density is presented also. Section IV summarizes the overall work.

\section{MODEL AND FORMULATION}

The systems under consideration are shown in Fig. 1, (a) the armchair GNRs, and (b) the zigzag GNRs. The GNRs are separated by two interfaces (denoted by two vertical dashed lines) into three parts: one central part (channel) and two electrodes (contacts), which is the common experimental setup of a transistor where a bias and a gate voltage, as well as a temperature difference between the two electrodes, are ready to be applied. The GNRs may be subjected to Rashba-type spin-orbit interaction due to gated substrate electric field, as well as other dephasing scattering processes. In what follows, we will adopt nonequilibrium Green function method to investigate effects of RSO and dephasing scattering processes on electronic and thermal electronic transport properties of such systems. The atomic tightbinding Hamiltonian for the GNRs is written as,

$$
H_{0}=\sum_{i, \sigma}\left(\epsilon_{i}+U_{i}\right) c_{i, \sigma}^{\dagger} c_{i, \sigma}+t \sum_{<i, j>, \sigma}\left(c_{i, \sigma}^{\dagger} c_{j, \sigma}+H . c .\right)
$$

where $\epsilon_{i}$ is the on site energy, $U_{i}$ is electric potential comes from the applied bias. $\sigma$ is electron spin operator. $t$ is electron hopping energy between neighboring carbon atoms, while the summation over $\langle i, j\rangle$ is restricted to the nearest-neighboring atoms. $c_{i, \sigma}\left(c_{i, \sigma}^{\dagger}\right)$ is the annihilation (creation) operator of a $2 p_{z}$ electron with spin $\sigma$ at site $i$. The Rashba 
spin-orbit interaction in two dimensional (2D) system is described by,

$$
H_{R}=\eta_{R}(\sigma \times \vec{k}) \cdot \hat{z}=\eta_{R}\left(\sigma_{x} k_{y}-\sigma_{y} k_{x}\right)
$$

where $\eta_{R}$ is Rashba interaction strength parameter. $\vec{k}=\left(k_{x}, k_{y}\right)$ is electron wave vector in the $2 \mathrm{D}$ plane, and $\hat{z}$ is unit vector perpendicular to the $2 \mathrm{D}$ plane. When Rashba spin orbit interaction is presented, the electrons traveling along $x$ axis should feel an effective magnetic field along $y, H_{R}=\sigma \cdot B_{\text {eff }}$ with $B_{\text {eff }}=\eta_{R}(\hat{z} \times \vec{k})$, and it is clear that $H_{R}$ will have opposite sign for electrons with opposite spin. We now try to map $H_{R}$ into a lattice model in the tight binding approximation. In low-energy electron transport or in long wavelength limit, $k a \approx \sin (k a)$, the RSO Hamiltonian Eq. (2) can be rewritten as,

$$
\begin{aligned}
H_{R} & =\eta_{R}\left(\sigma_{x} k_{y}-\sigma_{y} k_{x}\right) \approx \frac{\eta_{R}}{a}\left[\sigma_{x} \sin \left(k_{y} a\right)-\sigma_{y} \sin \left(k_{x} a\right)\right] \\
& =\frac{\eta_{R}}{2 i a} \sigma_{x}\left(e^{i k_{y} a}-e^{-i k_{y} a}\right)-\frac{\eta_{R}}{2 i a} \sigma_{y}\left(e^{i k_{x} a}-e^{-i k_{x} a}\right)
\end{aligned}
$$

It is noted that the hopping energy term $t$ in Hamiltonian Eq. (1) comes from terms like $t e^{ \pm i k_{x, y} a}$, according to atomic tight binding theory. Similarly, the RSO Hamiltonian Eq. (3) can be mapped to an atomic tight binding form by replacement of $t \mathrm{I} \rightarrow \mathrm{tI}+\left(\left(\eta_{\mathrm{R}} / 2 \mathrm{ia}\right) \sigma \times \hat{\mathrm{n}}\right) \cdot \hat{\mathrm{z}}$, where $\mathrm{I}$ is unit matrix in $2 \times 2$ spin space, and $\hat{n}$ the unit vector along $c-c$ bond in the nanoribbon plane. From Eqs. (1), (3) and Fig. (1), it is straightforward that the atomic Hamiltonian of the central graphene nanoribbon with RSO interaction, $H=H_{0}+H_{R}$, can be written in a block tridiagonal matrix form as,

$$
H=\left[\begin{array}{ccccc}
\alpha_{1} & \beta_{1} & 0 & 0 & 0 \\
\beta_{1}^{\dagger} & \alpha_{2} & \ldots & 0 & 0 \\
\vdots & \vdots & \vdots & \vdots & \vdots \\
0 & 0 & \ldots & \alpha_{N-1} & \beta_{N-1} \\
0 & 0 & 0 & \beta_{N-1}^{\dagger} & \alpha_{N}
\end{array}\right]
$$


where the diagonal submatrix $\alpha_{k}$ denotes for the isolated $k$ th layer of the central GNR channel, as indicated by dash-line rectangle in Fig. 1 , and the submatrices $\beta_{j}$ and $\beta_{j}^{\dagger}$ on the upper and lower diagonals denote for the interaction between two neighboring layers. $\alpha$ 's and $\beta$ 's are matrices of size $2 n \times 2 n$, with $n$ the number of carbon atoms in each layer and factor 2 comes from the spin freedom. $N$ is the number of layer units in the channel. It is very easy to write down submatrices $\alpha$ 's and $\beta$ 's according to the GNR crystal structure as shown in Fig. 1.

In NEGF theory, the retarded Green function $G$, which describes the central GNR channel and the connections to the two electrodes, is given as,

$$
G=\left[\left(E+i 0^{+}\right)-H-\Sigma_{L}-\Sigma_{R}-\Sigma_{s}\right]^{-1}
$$

where $E$ is quasiparticle energy measured with respect to the Fermi level $\mu$, and $0^{+}$is a positive infinitesimal number, which defining the "retarded" character of the Green function. The effect of the two contacts is included through the self-energy matrices appearing in Eq. (5), which are given by

$$
\Sigma_{L, R}=\beta_{L, R} g_{(L, R)} \beta_{L, R}^{\dagger}
$$

where $g_{L(R)}$ is surface Green function at the left (right) interface, and $\beta_{L(R)}$ being the interaction submatrix between the channel and left (right) contact, respectively. The surface Green function can be obtained from the following iterative equation,[26]

$$
\left.g_{L, R}^{s}=\left[(E+i \eta) I-\alpha_{L, R}^{s}-\tau_{L, R}^{s} g_{L, R}^{s+1} \tau_{L, R}^{s}\right]^{\dagger}\right]^{-1}
$$

where $\alpha_{L(R)}^{s}$ and $\tau_{L(R)}^{s}$ are layer Hamiltonian and interlayer interaction submatrices in the left (right) contact $(s=1,2, \cdots)$, and $g_{L(R)}^{s}$ stands for layer Green function, from the first layer 
at the interface $(s=1)$, to each inside layer into the left (right) contact. It is noted that Eq. (7) results in two self-contained iterative equations due to the longitudinal periodicity of the contact lattice, and these equations are solved numerically by a reasonable guess for initial surface Green function. Eventually, the two self-energy functions are calculated by Eq. (6). The last term in Eq. (5), $\Sigma_{s}$, is the self-energy matrix accounting for dephasing processes inside the central channel, for which we will adopt dephasing model the same as in Ref. [24], where it is made much more convenient for including dephasing processes in nonequilibrium Green function based quantum transport model. In the first order self-consistent Born approximation, $\Sigma_{s}$ is given as,

$$
\Sigma_{s}(i, j)=D(i, j) G(i, j)
$$

where $D(i, j)$ can be viewed as the correlation between the dephasing potential at the points $i$ and $j$ due to random fluctuations, or electron-electron interactions. Here for simplicity, we restrict our discussion to elastic dephasing where no energy is exchanged between the electrons and the dephasing source. Two different types of elastic dephasing are given by defining the matrix $D$ as follows, $D(i, j)=d_{m} \delta_{i j}$ for momentum relaxing, while $D(i, j)=d_{p}$ for momentum conserving. Here, $d_{m}$ and $d_{p}$ are constant factors representing the strength of dephasing processes with unit of $e V^{2}$. The momentum relaxing dephasing relaxes both phase and momentum, while the momentum conserving dephasing only relaxes phase, respectively. In other words, the former one affects both phase and momentum relaxation lengths, while the second one affects only phase relaxation length, and it is noted that real processes are usually somewhat between the two extremes of $d m$ and $d p$. 
Once the Hamiltonian and the self-energy functions are obtained, the retarded Green's function is ready to be calculated and all quantum transport quantities of interest can be obtained from the following set of NEGF equations, [26]

$$
\begin{gathered}
\Sigma^{i n}=\Sigma_{L}^{i n}+\Sigma_{R}^{i n}+\Sigma_{s}^{i n}=f_{L} \Gamma_{L}+f_{R} \Gamma_{R}+D(i, j) G^{n}(i, j) \\
A(E)=i\left[G(E)-G^{\dagger}(E)\right] \\
G^{n}(E)=G \Sigma^{i n} G^{\dagger} \\
T(E)=\operatorname{Tr}\left(\Sigma^{i n} A-\Gamma G^{n}\right) /\left(f_{L}-f_{R}\right)
\end{gathered}
$$

where $f_{L(R)}=1 /\left[e^{\frac{E-\mu_{L(R)}}{k_{B} T_{L(R)}}}+1\right]$ is Fermi-Dirac distribution function of electron in the left (right) contact, which is assumed to be equilibrium with a electric and a thermal reservoir, $\mu_{L(R)}$ and $T_{L(R)}$, respectively. $\quad \Sigma^{i n}$ is known as inscattering function, with $\Gamma_{L(R)}=i\left[\Sigma_{L(R)}-\Sigma_{L(R)}^{\dagger}\right]$ describes the energy level broadening inside the central channel due to the left (right) contact. $G^{n}(E)$ and $A(E)$ are correlation function and spectral function in NEGF theory, whose diagonal elements are the electron density and the local density of state at the corresponding site, respectively. It is noted that both $G^{n}(E)$ and $A(E)$ are spin resolved and we can get nontrivial spin density at each site of the nanoribbon when the RSO interaction is presented. $T(E)$ is electronic conductance. It is noted that the above equations requiring an iterative self-consistent calculation in general. The charge and thermal current is given as, $[27]$

$$
\begin{gathered}
J_{e}=\int \frac{d E}{2 \pi} \frac{q}{\hbar} T(E)\left[f_{L}(E)-f_{R}(E)\right] \\
J_{Q}=\int \frac{d E}{2 \pi} \frac{E-\mu}{\hbar} T(E)\left[f_{L}(E)-f_{R}(E)\right]
\end{gathered}
$$


It is noted that the charge and thermal currents are driven by the difference between $f_{L}$ and $f_{R}$, which may be resulted from either voltage or temperature differences, while the particular behavior depends on $T(E)$. We can linearized Eqs. (13) and (14) to obtain an expression for both electronic and thermal current in terms of voltage and temperature differences as,

$$
\begin{gathered}
J_{e}=L_{0}\left(V_{L}-V_{R}\right)+L_{1}\left(T_{L}-T_{R}\right) \\
J_{Q} / T=L_{1}\left(V_{L}-V_{R}\right)+L_{2}\left(T_{L}-T_{R}\right)
\end{gathered}
$$

with

$$
L_{m}=\int d E T(E)\left(\frac{E-\mu}{q T}\right)^{m} \frac{\partial f(E)}{\partial E}
$$

The well known Seebeck coefficient $S$ and thermoelectric figure of merit $Z T$ are given as,

$$
\begin{gathered}
S=-\frac{L_{1}}{L_{0}} \\
Z T=\frac{S^{2} G_{e} T}{\kappa_{e}}
\end{gathered}
$$

with $G_{e}=L_{0}$ is the total electrical conductance and $\kappa_{e}=L_{2} T-L_{1}^{2} T / L_{0}$ is the electron thermal conductance. The denominator of $Z T$ is heat conductance contributed by electrons, and it is noted that a main part of heat conductance comes from phonon transport is not considered here, which is very different from the electronic transport properties. Therefore $Z T$ is not a good index for the thermoelectric property in the present paper. The practical importance of thermoelectric effects lies in the possibility of converting waste heat energy into electricity. The numerator of $Z T$ is called power factor $P=S^{2} G_{e}$, which is the amount of power that could be generated from a given temperature difference, and it is known as 
one of the standard figures of merit for thermoelectric materials, and the higher $P$ the better performance of the material.

\section{RESULTS AND DISCUSSIONS}

We present calculation results now. At first, we focus on effects of Rashba spinorbit interaction and suppress the dephasing term $\Sigma_{s}$ in Eq. (5). In Fig. 2, we plot the conductance $T(E)$ of armchair (a) and zigzag (b) GNRs as a function of quasiparticle energy $E$, for different values of the RSO interaction strength $\eta_{R}$ as indicated in the figures. It is noted that $\eta_{R}$ is turnable by the substrate gated electric field, which is a common setup in transistors. In calculation, the on site energy $\epsilon_{i}$ and the hopping energy $t$ are set to be zero and $-2.7 \mathrm{eV}$ according to experimental data, respectively. The width and length of the central armchair GNRs are $1.845 \times 10^{-9} \mathrm{~m}$, and $2.414 \times 10^{-9} \mathrm{~m}$, respectively, which is used throughout the paper. We shall point out that we are considering transport regime close to ballistic with RSO and elastic dephasing scattering processes. In this case, the conductance depends on the density of states per unit length along the current direction, in general. For 2-D graphene nanoribbons, the density of states is proportional to the area (width times length), and therefore the conductance will be proportional to the width and have no explicit dependence on the length. It is found that the conductance is suppressed with increasing $\eta_{R}$. and at the same time, the larger of the quasiparticle energy $E$, the much suppression of $T(E)$ is got. Physically, a remarkable consequence of Rashba spin-orbit interaction is the splitting of the original Dirac point caused by crossing of conduction and valence bands, and 
consequently modifies the electron energy band structure of GNRs and suppression of the conductance.

In Fig. 3, we compare thermoelectric behaviors (a) charge current $J e$, (b) thermal current $J_{Q}$, (c) Seebeck coefficient $S$, and (d) the figure of merit power factor $P$ of zigzag and armchair GNRs, as a function of the Fermi level $\mu$. The voltage applied between left and right electrodes is set to be $0.1 \mathrm{~V}$, and temperatures of $k_{B} T_{L}=0.01 \mathrm{eV}$ and $k_{B} T_{R}=0.011 \mathrm{eV}$, respectively. The charge current (Fig. 3a) is a simple integration of conductance and effective fermi function difference (Eq. 13), and therefore it is similar to the conductance (Fig. 2). While according to Eq. 14, quasiparticles with energy below and above the fermi level $\mu$ contribute oppositely to the thermal current $J_{Q}$, and therefore $J_{Q}$ equals almost zero when $\mu$ locates at the conductance plateaus and peaks itself when $\mu$ is at the transitions between two plateaus, as shown in Fig. 3b. For the GNRs width we used here for calculation, the electrical conductance is nonzero for zigzag, while it is vanishing for armchair ones, for quasiparticle energies around the fermi level, respectively. As a result, the vanishing conductance which appears in the denominator of Seebeck coefficient $S$, Eq. 18, will mathematically lead to large peaks of $S$ as shown in Fig. 3c. We should point out that such large peak values of $S$ is physically meaningless because there will be no thermal current if there is no electrical current. For clarity and comparison, we will neglect this part and the remaining curves are shown in the inset in Fig. 3c. It is seen that both Seebeck coefficient $S$ and power factor $P$ have peaks, which means better performance of thermoelectricity, for particular fermi levels around the transitions between adjacent conductance plateaus, than elsewhere. 
In Fig. 4, we have calculated the charge current $J e(a)$, heat current $J_{Q}$ (b), Seebeck coefficient $S$ (c), and the power factor $P$ (d) as a function of the Fermi level $\mu$, for armchair GNRs with different values of the Rashba interaction strength $\eta_{R}$. Similar to the conductance (Fig. 2), the charge current $J e$ is suppressed with increasing strength of $\eta_{R}$, while the peak position of thermal current $J_{Q}$ varies with $\eta_{R}$, which is resulted from modification of the conductance. It is noted that the thermal currents will be negative during ranges of negative slope of $T(E)$ where thermal contribution from electrons with energy below $\mu$ exceeds those with energy above $\mu$, as shown in Fig. 4b. The negative thermal current consequently leads to sign change of Seebeck coefficient $S$ (Fig. 4c), as well as reduction of power factor (Fig. 4d). Plotting in Fig. 5 is the same as Fig. 4 except for zigzag GNRs, and similar behaviors are observed. It is shown that the Rashba spin orbit interaction will reduce the performance of thermoelectric properties of graphene nanoribbons, rather than improve it.

As stated above, it is convenient to calculate electron density and density of states at each site of GNRs in the framework of NEGF theory, which are given by the correlation function $G_{n}(E)$ and spectral function $A(E)$, respectively. The ratio $\mu_{\uparrow}(E, i)=G^{n}(E ; 2 i-1,2 i-$ 1)/A(E;2i-1,2i-1) tells us the effective occupation factor throughout the central channel, which specifically corresponds to a local electrochemical potential for up-spin electrons of site $i$, while $\mu_{\downarrow}(E, i)=G^{n}(E ; 2 i, 2 i) / A(E ; 2 i, 2 i)$ for down-spin electrons, at quasiparticle energy $E$. Here $i=1, \cdots, n N$ with $n N$ the total number of carbon atoms in the GNRs. Therefore it is ready to present spin density induced by RSO, which is given in Fig. 6 for armchair, and in Fig. 7 for zigzag GNRs, for two different strength of RSO as indicated, 
respectively. The color bar on each site represents relative spin density, which is defined as, $D_{\sigma}=\left[\mu_{\uparrow}(E, i)-\mu_{\downarrow}(E, i)\right] /\left[\mu_{\uparrow}(E, i)+\mu_{\downarrow}(E, i)\right]$. Spin fluctuations induced by Rashba spin orbit interaction, along both transverse and longitude directions, are clearly presented.

We now turn on $\Sigma_{s}$ in Eq. (5) to study effects of dephasing processes. We plot (a) charge current $J e$, (b) thermal current $J_{Q}$, (c) Seebeck coefficient $S$, and (d) power factor $P$ as a function of the Fermi level $\mu$, for armchair GNRs with momentum relaxing dephasing (Fig. 8) and momentum conserving dephasing (Fig. 9) processes, respectively. It is found that the charge currents $J e$ are suppressed generally by such dephasing scattering processes, while the stepwise characteristics of $J_{e}$ conserved for momentum relaxing dephasing cases (Fig. 8a) and it is smoothed out gradually for momentum conserving dephasing ones (Fig. 9a). Comparing the other three figures of Figs. 8 and 9, it is shown that the momentum relaxing $(d m)$ and conserving $(d p)$ scattering processes have very similar and slight effects on the present electron-related thermal transport properties of GNRs. Plottings in Figs. 10 and 11 are the same as in Figs. 8 and 9, except for zigzag GNRs, and similar effects of dephasing processes was shown. We shall point out that such dephasing processes may be closely related to phonon thermal transport which needs further investigation.

\section{SUMMARY}

In summary, we have theoretically studied thermoelectric transport properties of zigzag and armchair GNRs, with presence of Rashba spin orbit interaction and dephasing scattering processes. Detailed behaviors of charge current and thermal current, as well as ther- 
moelectric properties of Seebeck coefficient and the figure of merit thermal power factor are reported. It is found that the charge and thermal currents of GNRs are reduced, and thermoelectric properties been suppressed, in general, with increasing of the strength of RSO interaction. It is shown that the two models of dephasing scattering processes have slight effects on electron-related thermal transport properties of GNRs. In addition, RSO interaction induced spin split and spin density is calculated, and spin density fluctuation along both transverse and longitude directions of the GNRs is shown clearly.

\section{Acknowledgments}

This work is supported by the National Science Foundation of China under grant No. 10704062 and No. 11275162. 
[1] E. B. Sonin, Phys. Rev. B 79, 195438 (2009).

[2] M. I. Katsnelson, K. S. Novoselov, and A. K. Geim, Nature Physics 2, 620 (2006).

[3] A. K. Geim and K. S. Novoselov, Nature materials 6, 183 (2007).

[4] K. S. Novoselov, A. K. Geim, S. V. Morozov, D. Jiang, Y. Zhang, S. V. Dubonos, I. V. Grigorieva, and A. A. Firsov, Science 306, 666 (2004).

[5] M. Wilson, Phys. Today 59(1), 21 (2006).

[6] B. Huang, Q. Yan, Z. Li, and W. Duan, Front. Phys. China 4(3), 269 (2009).

[7] A. Dato, V. Radmilovic, Z. Lee, J. Phillips, and M. Frenklach, Nano Lett. 8, 2012 (2008).

[8] A. Reina, X. Jia, J. Ho, D. Nezich, H. Son, V. Bulovic, M. S. Dresselhaus, and J. Kong, Nano Lett. 9, 30 (2009).

[9] Keun Soo Kim, Y. Zhao, H. Jang, S. Y. Lee, J. M. Kim, Kwang S. Kim, J. -H. Ahn, P. Kim, J. -Y. Choi, B. H. Hong, Nature (London) 457, 706 (2009).

[10] S. Stankovich, D. A. Dikin, G. H. B. Dommett, K. M. Kohlhaas, E. J. Zimney, E. A. Stach, R. D. Piner, S. T. Nguyen, and R. S. Ruoff, Nature (London) 442, 282 (2006).

[11] H. C. Schniepp, J. -L. Li, M. J. McAllister, H. Sai, M. Herrera-Alonso, D. H. Adamson, R. K. Prudhomme, R. Car, D. A. Saville, and I. A. Aksay, J. Phys. Chem. B 110, 8535 (2006).

[12] M. Wierzbowska, A. Dominiak, and G. Pizzi, 2D Materials 1, 035002 (2014).

[13] M. Alomar and D. Sanchez, Phys. Rev. B 89, 115422 (2014).

[14] M. Inglot, V. K. Dugaev, and J. Barna, Phys. Rev. B 92, 085418 (2015).

[15] J. H. Seol, I. Jo, A. L. Moore, L. Lindsay, Z. H. Aitken, M. T. Pettes, X. Li, Z. Yao, R. Huang, D. Broido, N. Mingo, R. S. Ruoff, L. Shi, Science 328, 213 (2010).

[16] D. Dragoman and M. Dragoman, Appl. Phys. Lett. 91, 203116 (2007).

[17] T. Stauber, N. M. R. Peres, and F. Guinea, Phys. Rev. B 76, 205423 (2007).

[18] M. Inglot, A. Dyrdal, V. K. Dugaev, and J. Barnas, Phys. Rev. B 91, 115410 (2015).

[19] C. L. Kane and E. J. Mele, Phys. Rev. Lett. 95, 146802 (2005).

[20] C. L. Kane and E. J. Mele, Phys. Rev. Lett. 95, 226801 (2005).

[21] Ralph van Gelderen and C. Morais Smith, Phys. Rev. B 81, 125435 (2010).

[22] Y. S. Dedkov, M. Fonin, U. Rudiger, and C. Laubschat, Phys. Rev. Lett. 100, 107602 (2008).

[23] A. Varykhalov, J. Sanchez-Barriga, A. M. Shikin, C. Biswas, E. Vescovo, A. Rybkin, D. 
Marchenko, and O. Rader, Phys. Rev. Lett. 101, 157601 (2008).

[24] Roksana Golizadeh-Mojarad and S. Datta, Phys. Rev. B 75, 081301(R) (2007).

[25] M. Buttiker, IBM J. Res. Dev. 32, 72 (1988).

[26] S. Datta, Electronic Trnsport in Mesoscopic Systems, Cambridge University Press, Cambridge, (1995).

[27] M. Paulsson and S. Datta, Phys. Rev. B 67, 241403 (2003). 


\section{FIGURE CAPTIONS}

Fig. 1 Sketch of (a) armchair and (b) zigzag graphene nanoribbons (GNRs). Vertical dashed lines separate the GNRs into three parts: two contact electrodes and one central channel.

Fig. 2 The conductance $T(E)$ of (a) armchair and (b) zigzag GNRs, as a function of quasiparticle energy $E$, for different $\eta_{R}$ as indicated.

Fig. 3 The charge current (a) Je, thermal current (b) $J q$, (c) Seebeck coefficient $S$, and (d) the figure of merit power factor $P$ as a function of the Fermi level $\mu$, for armchair GNRs (solid) and zigzag (dotted), respectively.

Fig. 4 The charge current (a) Je, thermal current (b) $J q$, (c) Seebeck coefficient $S$, and (d) the figure of merit power factor $P$ as a function of the Fermi level $\mu$, for armchair GNRs. With different strength of RSO interaction $\eta_{R}$,

Fig. 5 The same as Fig. 4, except for zigzag GNRs.

Fig. 6 Spin density at each site of armchair graphene nanoribbon, for (a) $\eta_{R}=2 \times$ $10^{-10} \mathrm{eV} \cdot \mathrm{m}$ and (b) $\eta_{R}=4 \times 10^{-10} \mathrm{eV} \cdot \mathrm{m}$, with $E=1.5 \mathrm{eV}$.

Fig. 7 The same as Fig. 6 except for zigzag graphene nanoribbons. 
Fig. 8 The charge current (a) Je, thermal current (b) $J q$, (c) Seebeck coefficient $S$, and (d) the figure of merit power factor $P$ as a function of the Fermi level $\mu$, for armchair GNRs. With different strength $d_{m}\left(e V^{2}\right)$ of momentum relaxing dephasing process.

Fig. 9 The same as Fig. 8, except for different strength $d_{p}\left(e V^{2}\right)$ of momentum conserving dephasing process.

Fig. 10 The same as Fig. 8, except for zigzag GNRs.

Fig. 11 The same as Fig. 9, except for zigzag GNRs. 
Fig.1(a)

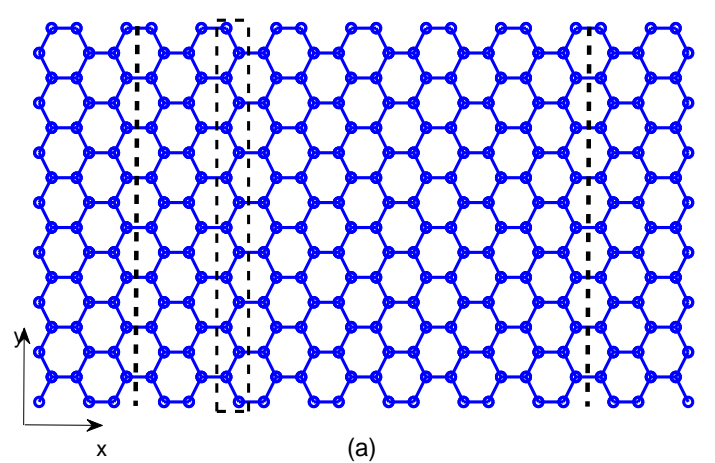

Fig.1(b)

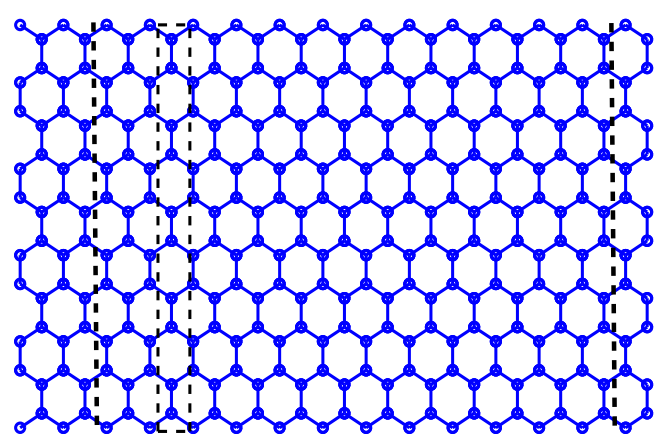

(b)

Fig.2
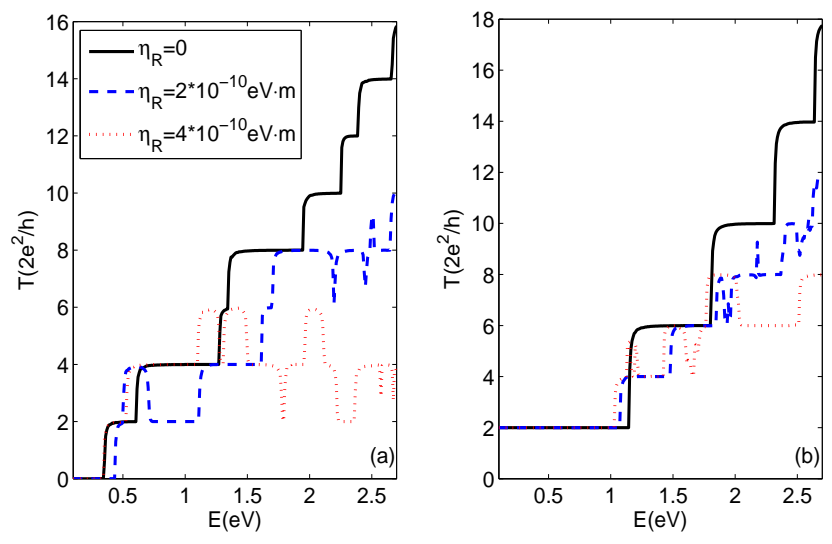
Fig.3
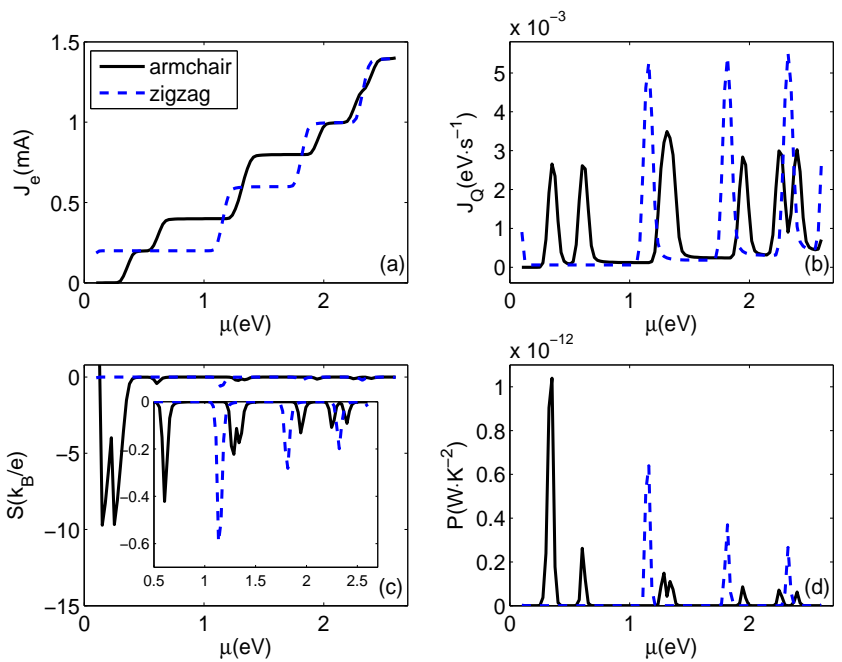

Fig.4
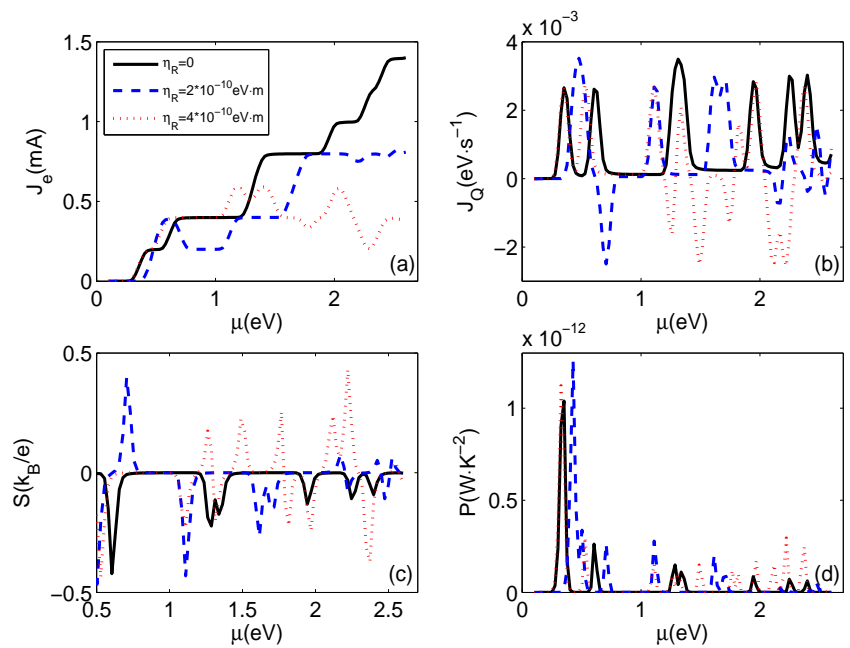

Fig.5
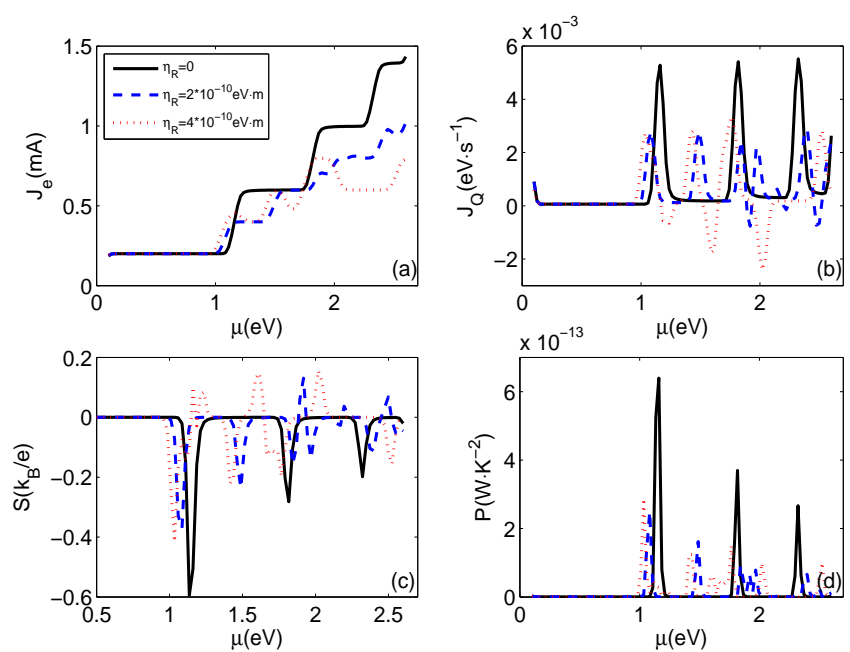
Fig.6(a)

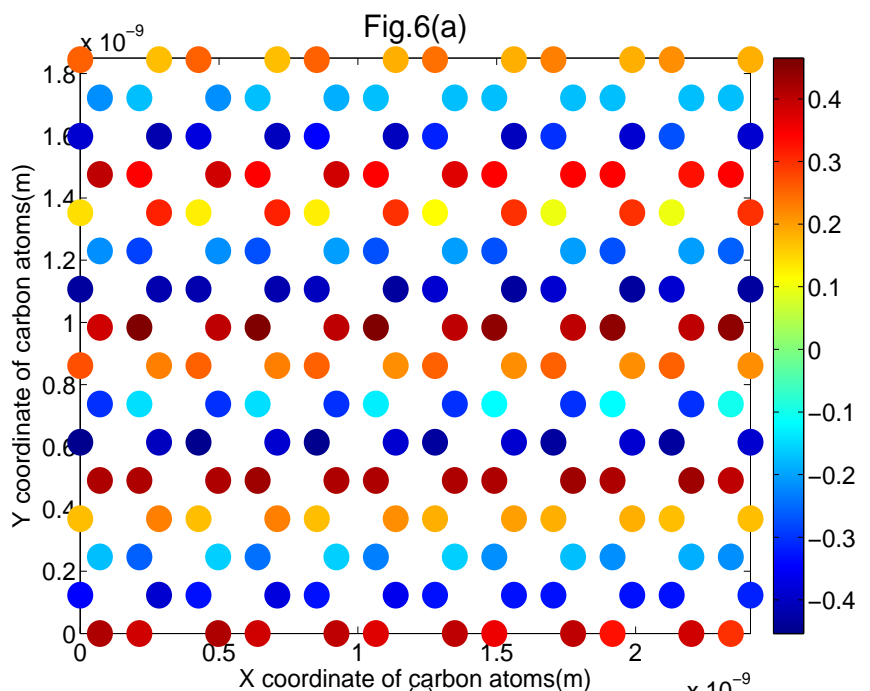

$\mathrm{X}$ coordinate of carbon atoms $(\mathrm{m}) \times 10^{-9}$
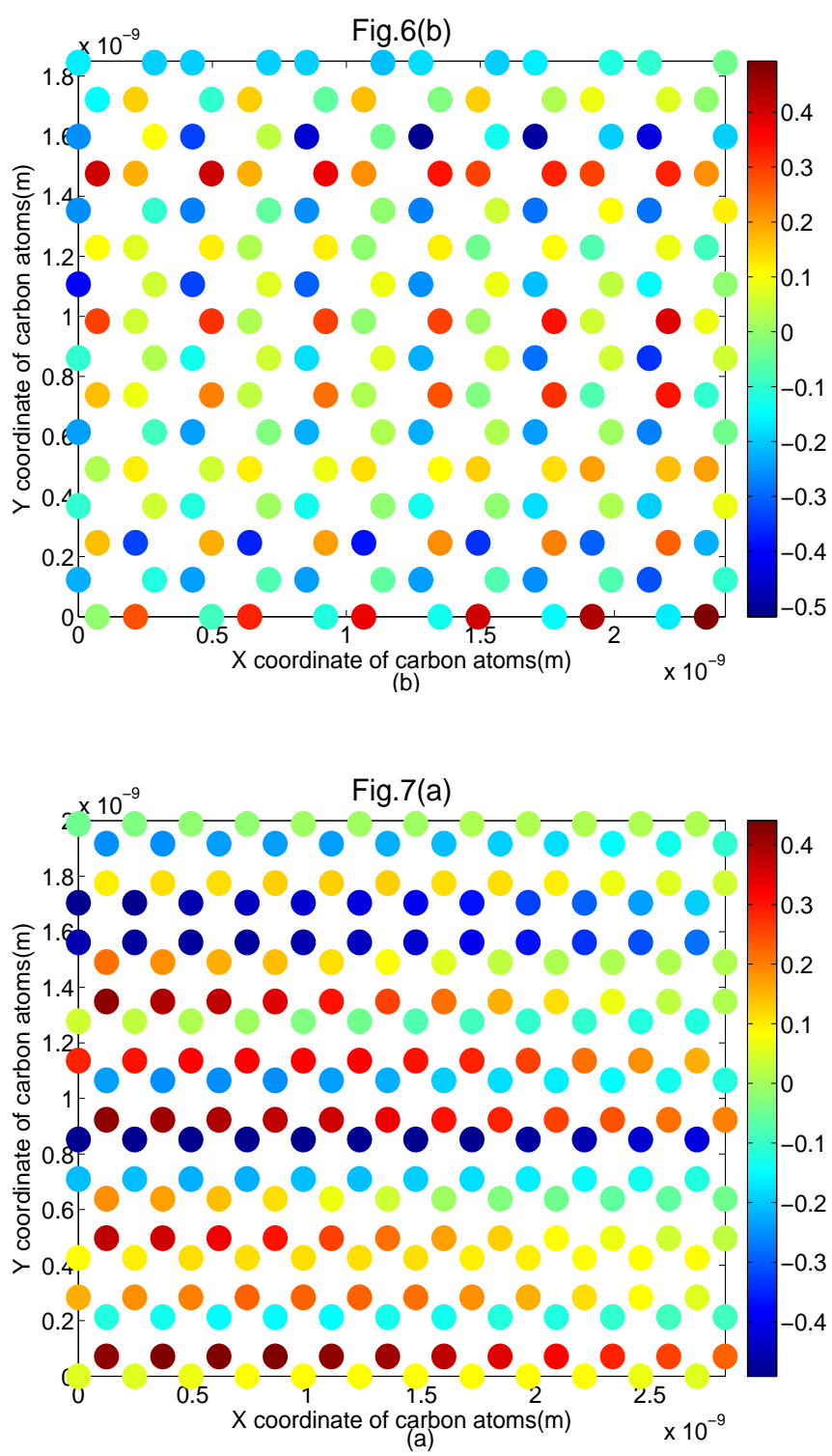
Fig.7(b)
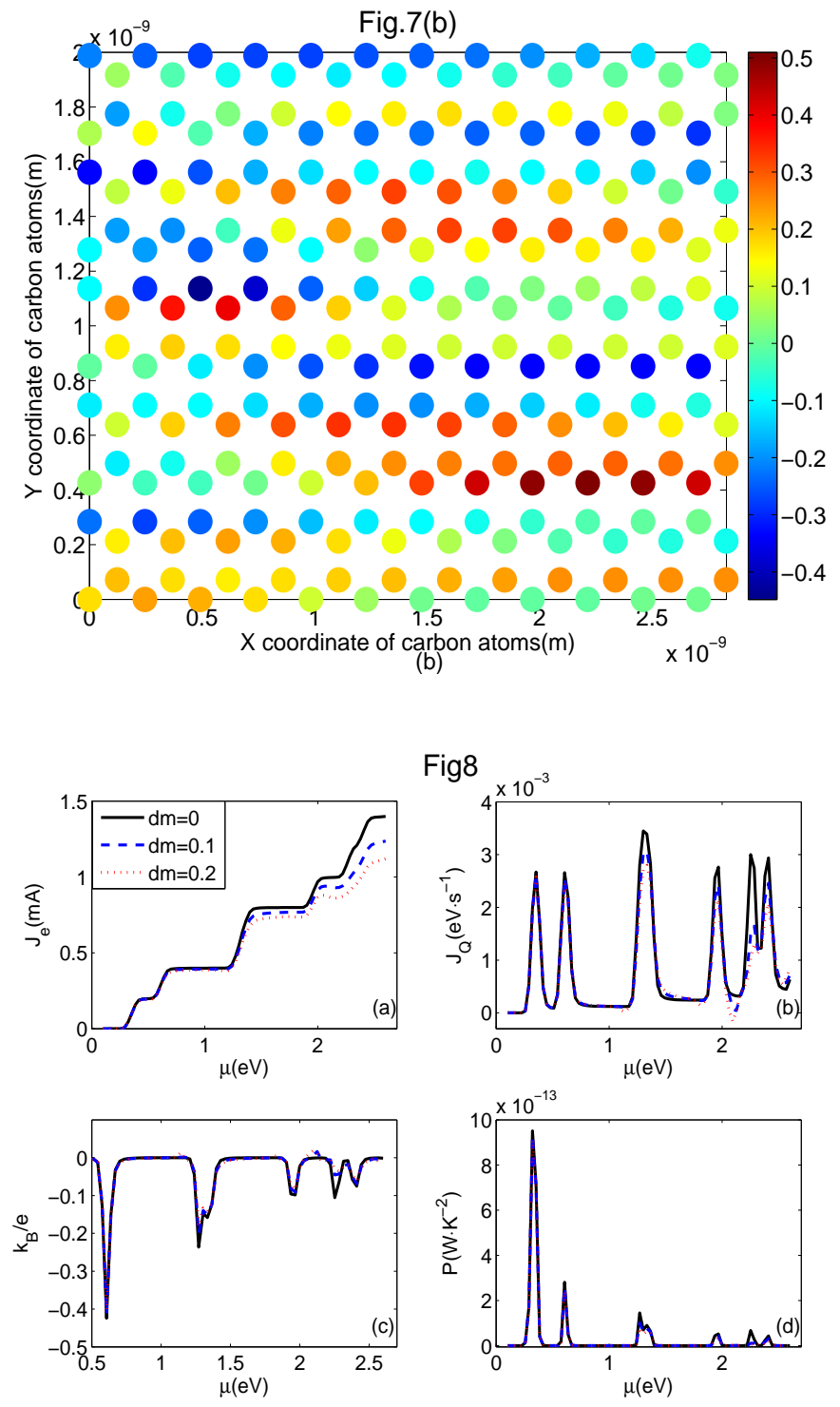

Fig. 9
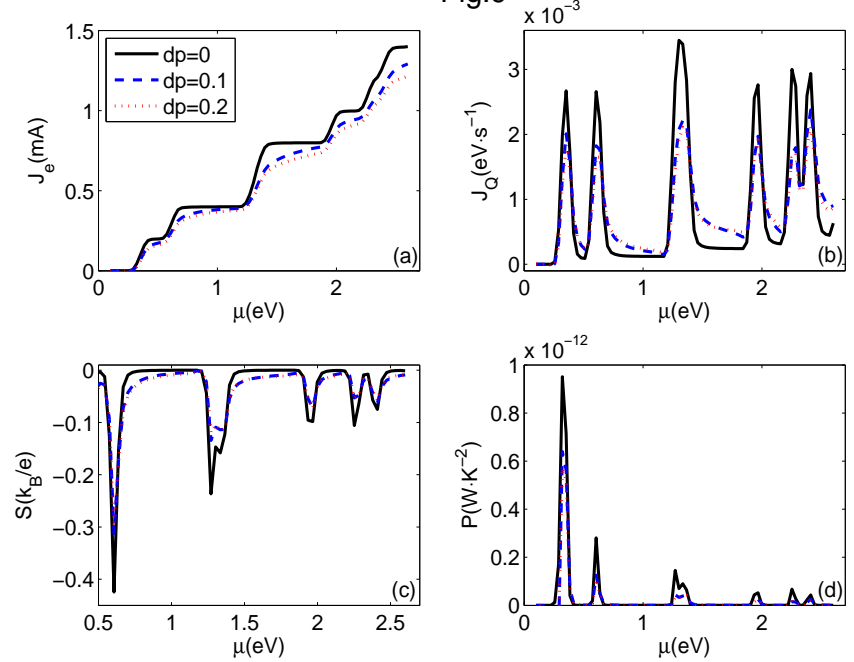
Fig. $10 \times 10^{-3}$
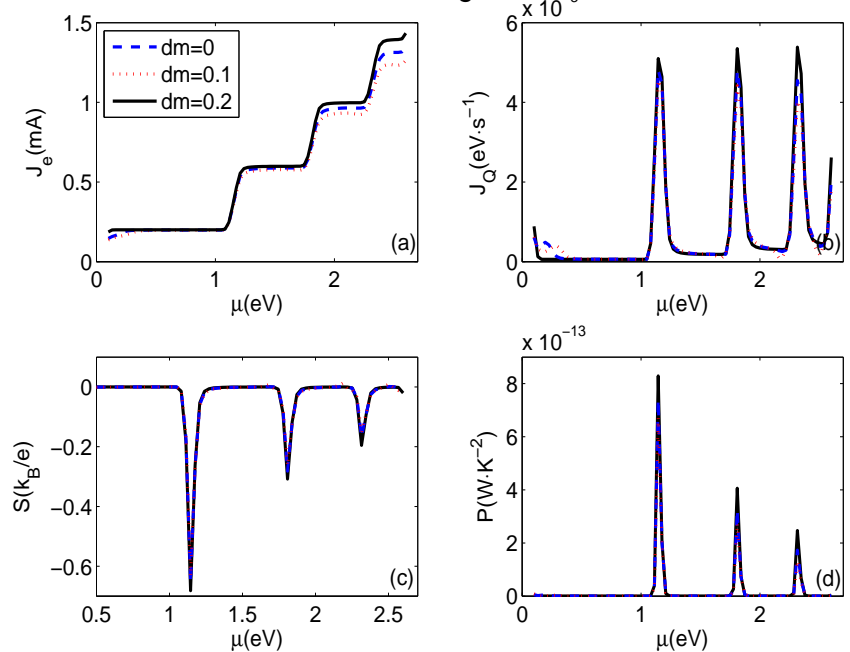

Fig. 11
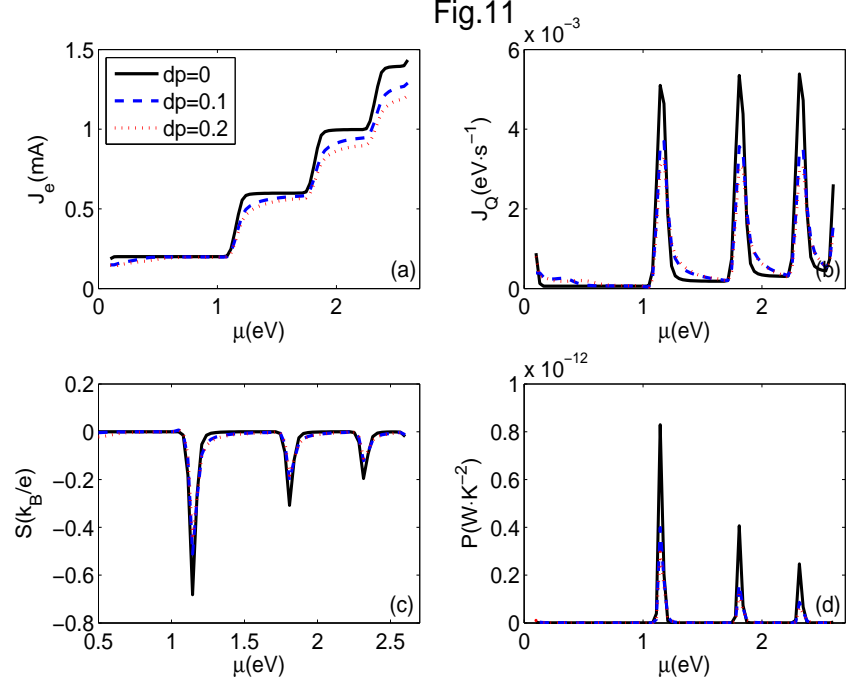